\title{
Antibiotic Prophylaxis against Surgical Site Infection after Open Hernia Surgery: A Systematic Review and Meta-Analysis
}

\author{
Lolwah Al Riyees $^{a}$ Wedad Al Madani ${ }^{b}$ Nistren Firwanac Hanan H. Balkhy ${ }^{d}$ \\ Mazen Ferwana ${ }^{e, f, g}$ Abdullah Alkhudhayrih \\ aKing Abdullah International Medical Research Center, King Saud bin Abdulaziz University for Health Sciences, \\ Consultant of General Surgery, King Abdulaziz Medical City, Ministry of National Guard Health Affairs, Riyadh, Kingdom \\ of Saudi Arabia; 'benior Epidemiologist, National Center for Evidence-Based Health Practice, Saudi Health Council, \\ Riyadh, Kingdom of Saudi Arabia; 'Executive Master of Health Informatics, University of Toronto, Toronto, ON, Canada; \\ dWorld Health Organization, Geneva, Switzerland; eProfessor of Family Medicine, King Abdullah International Medical \\ Research Center, King Saud bin Abdulaziz University for Health Sciences, Riyadh, Kingdom of Saudi Arabia; ${ }^{f} \mathrm{Co}$-director, \\ National and Gulf Center for Evidence- Based Health Practice, Riyadh, Kingdom of Saudi Arabia; ${ }^{9}$ Consultant, Family \\ Medicine and Primary Healthcare Department, King Abdulaziz Medical City, Ministry of National Guard Health Affairs, \\ Riyadh, Kingdom of Saudi Arabia; ' hinistry of Health, Riyadh, Kingdom of Saudi Arabia
}

\section{Keywords}

Prophylactic antibiotics · Hernia repair · Surgical site

infection · Systematic review · Meta-analysis

\begin{abstract}
Objective: The role of antibiotic prophylaxis (AP) in the prevention of surgical site infection (SSI) after hernia repair is debated. We conducted this systematic review and metaanalysis to assess the evidence on the value of prophylactic antibiotics in reducing the risks of SSI after open hernia surgery. Methods: We ran an online and manual search to identify relevant randomized controlled trials that compared prophylactic antibiotics to nonantibiotic controls in patients undergoing open surgical hernia repair. Data on SSI risk were extracted and pooled as risk ratios (RRs) with 95\% confidence intervals (95\% Cls), using RevMan software. We further used the Cochrane risk of bias tool and GRADE assessment to evaluate the quality of generated evidence. Results: Twenty-nine studies ( $N=8,616$ patients) were included in the current analysis. Antibiotic prophylaxis reduced the risk of SSI in open
\end{abstract} \\ Karger'"}

hernia repair patients $(R R=0.65,95 \% C l=0.53,0.79)$. Subgroup analysis showed a significant benefit for antibiotics in mesh repair patients $(\mathrm{RR}=0.60,95 \% \mathrm{Cl}=0.48,0.76)$ yet no significant difference in SSI risk after herniorrhaphy (RR = $0.86,95 \% \mathrm{Cl}=0.54,1.36)$. In addition, AP was associated with a significant reduction in superficial SSI risk (RR $=0.56,95 \%$ $\mathrm{Cl}=0.43,0.72)$ but not deep SSI (RR $=0.70,95 \% \mathrm{Cl}=0.30$, 1.62). Further analysis showed a significant reduction in SSI risk with amoxicillin/clavulanic acid and cefazolin but not with cefuroxime. Conclusion: The present meta-analysis suggests that AP is beneficial prior to open mesh hernia repair. However, the quality of evidence was low, and further welldesigned trials are needed.

(c) 2021 S. Karger AG, Basel

\section{Introduction}

Worldwide, almost 20 million hernia surgeries are performed each year. Most of these procedures are to repair inguinal hernias (IH) [1], and mesh repair (hernio-
Correspondence to:

Mazen Ferwana, mazenferwana@gmail.com 
plasty) is used in $90 \%$ of these cases [2]. Hernia surgery is generally considered to be a clean procedure. The rate of surgical site infections (SSIs) after hernia repair varies widely according to the type of center, type of hernia, and surgical technique but can be as low as 1-2\% [3]. Among other factors, SSI after hernia repair may predispose patients to hernia recurrence [4].

The appropriateness of the use of antibiotic prophylaxis (AP) before hernia surgery is debated. Earlier retrospective and prospective datasets have reported different practices and results [5-7]. The European Hernia Society does not recommend the routine use of AP in low-risk patients [8]. Several prospective randomized trials have explored the effectiveness of AP prior to hernia repair surgery. Some of these studies reported significant benefits for antibiotic use before surgery, while other studies failed to find such benefit [9-37].

In this meta-analysis, we analyzed the best available evidence examining the role of AP in preventing SSI after hernia repair. To that end, we compared the incidence of SSI in patients undergoing open hernia repair receiving prophylactic antibiotics versus nonantibiotic controls.

\section{Methods}

We performed this systematic review in compliance with the Cochrane handbook for systematic reviews of intervention [38] and reported it in compliance with the PRISMA checklist (see online suppl. Table 1; for all online suppl. material, see www. karger.com/doi/10.1159/000517404) [39].

\section{Literature Search}

We searched the following online databases to find randomized controlled trials (RCTs): Medline, Cochrane central library, EMBASE, CINAHL, ClinicalTrials.gov, and the World Health Organization search engines with no language restriction. The initial search was performed in May 2017; another updated search was performed in August 2020. We also screened the references lists of the included studies and published systematic reviews for all relevant studies that evaluated the benefits of prophylactic antibiotics in patients undergoing hernia repair operations. The search terms used were ["Hernia OR, Herniation OR, Herniotomy OR, Hernia repair” AND “Antibacterial agents OR, Antibiotic OR, Anti-bacterial OR, Clindamycin OR, Cefuroxime OR, Ceftazidime OR, Ofloxacin OR, Levofloxacin OR, Azithromycin OR, Sulbactam OR, Ampicillin OR, Mezlocillin OR, Oxacillin OR, Vancomycin OR, Tobramycin OR, Ciprofloxacin OR, Cefazolin OR, Gentamicin OR, Metronidazole OR, Neomycin OR, Doxycycline" AND, "Surgical site infection OR, Surgical wound infection OR, Surgical wound dehiscence OR, Infection control OR, Wound complication"]. MeSH terms and text key words were used.

Eligibility Criteria and Screening

We included RCTs that matched the following criteria: (1) population: adult patients undergoing open IH surgery, regardless elective or emergency, (2) intervention: prophylactic antibiotics, (3) control: patients may have received placebo, saline, multivitamins, or other nonantibiotic controls, (4) outcome: SSI (both superficial and deep SSI). Two reviewers (A.A. and N.F.) independently evaluated the title, abstract, and (if needed) full text of relevant studies for inclusion and exclusion criteria. Any discrepancies were resolved by consensus.

\section{Data Extraction and ROB Assessment}

Two researchers (W.M. and N.F.) independently extracted data using a special extraction form, and any discrepancies were resolved by discussion. The extracted data included the following: inclusion and exclusion criteria, setting, type of repair, type of antibiotic, SSI definition, and incidence. Two authors (M.F. and A.A.) independently assessed the relevant articles for the risk of bias (ROB) using the Cochrane Collaboration Criteria (to assess for selection bias, performance bias, reporting bias, attrition bias, and other sources of bias) [40]. We then used the GRADE quality of evidence assessment to assess the certainty of evidence of analyzed outcomes [41].

\section{Statistical Analysis}

The statistical analysis was performed using Review Manager software (RevMan version 5.3, Copenhagen: The Nordic Cochrane Centre, The Cochrane Collaboration, 2012). Meta-analysis was conducted to assess the primary outcome of SSI. Risk ratios (RRs) and $95 \%$ confidence intervals (95\% CIs) were calculated for pooling up the results of different studies, using Mantel-Haenszel fixed effects model analysis. Heterogeneity was explored using conventional Cochrane's $Q$ test and $I^{2}$ test. The $p$ value of the $Q$ test was considered significant when it was $\leq 0.10$. Publication bias was assessed by visually inspecting the funnel plot produced using Review Manager. We performed subgroup analyses by type of antibiotic and type of SSI.

\section{Results}

\section{Literature Search Results}

The initial search identified 5,629 related articles. The study selection process is shown in Figure 1, including the justification for exclusion. Only 29 RCTs met our inclusion criteria in which 8,616 patients participated (shown in Fig. 1) [9-37]. Of these participants, 4,718 patients received AP before surgery, compared with 3,898 patients who did not. Two studies $[17,26]$ compared different classes of antibiotics. Table 1 shows the characteristics of the included studies.

\section{Characteristics of the Included Studies}

Surgical Procedures

Nineteen studies included only patients who underwent open mesh repair of their IH. Two studies included patients who underwent only anatomic groin (femoral and inguinal) herniorrhaphy, both studies excluded mesh 
Fig. 1. PRISMA flow diagram showing the study selection process.

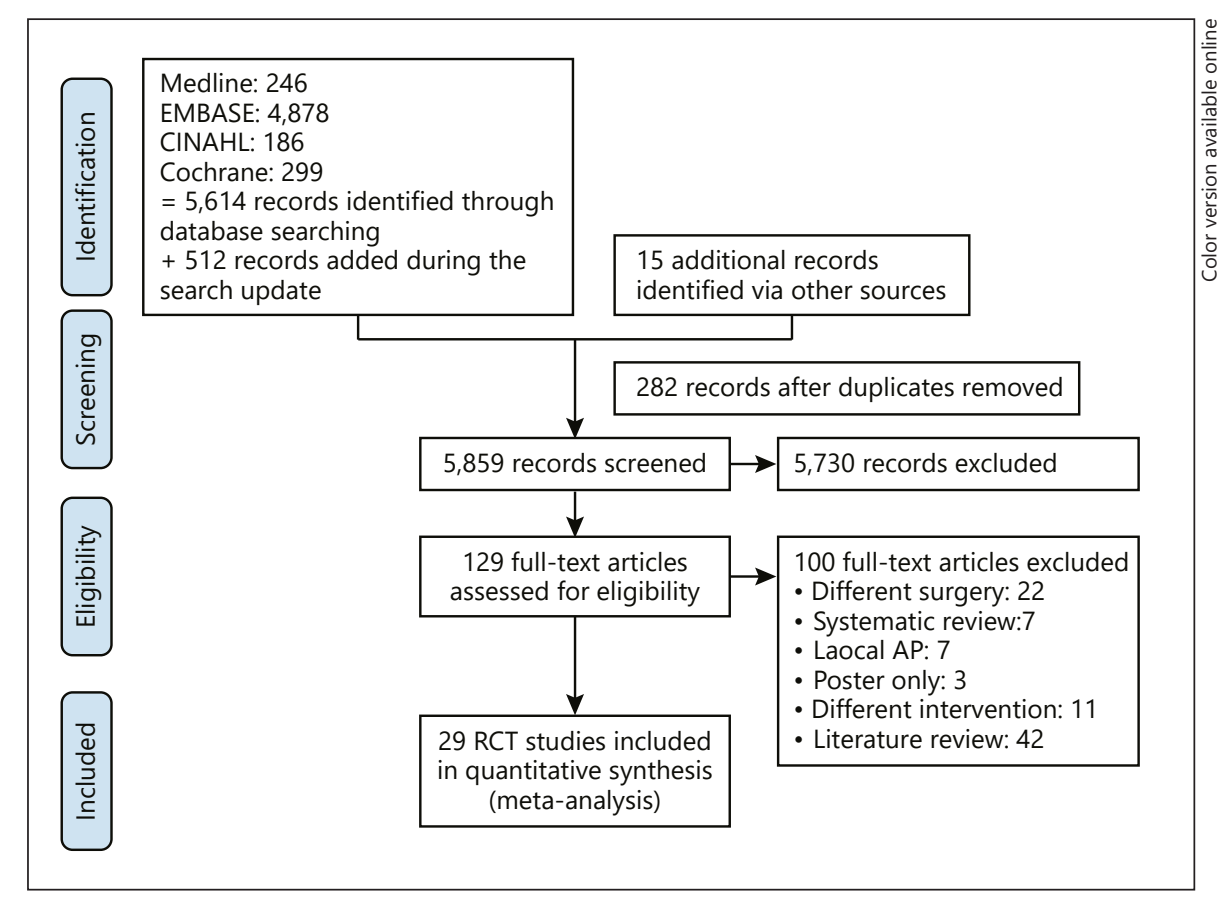

repair. The study by Taylor et al. included patients who had groin (inguinal and femoral) hernias repaired either by mesh or herniorrhaphy.

\section{Antibiotic Prophylaxis}

The included studied assessed 12 antibiotics belonging to 4 different classes (penicillins, cephalosporins, quinolones, and macrolides). Participants in all control groups took identically shaped placebos.

\section{ROB Assessment}

The risk of selection bias was high/unclear in about half of the included studies. Only 12 studies performed adequate blinding of the participants, and only 11 studies performed adequate blinding of outcome assessors. The majority of included studies had low risks of attrition and reporting biases. Figure 2 shows ROB items for each study. The results of GRADE assessment are illustrated in online suppl. Table 2.

\section{Overall SSI Rate}

The overall rate of SSI after hernia surgery was $4.27 \%$. Antibiotic prophylaxis was associated with an overall significant reduction in the SSI rate $(\mathrm{RR}=0.65,95 \% \mathrm{CI}=$ $0.53,0.79, p<0.0001 ; 28$ studies; $N=8,221$; low quality of evidence) compared to the control group. Subgroup analysis based on hernia surgery type showed that in hernio- plasty, AP was associated with significantly reduced SSI risk $(\mathrm{RR}=0.60,95 \% \mathrm{CI}=0.48,0.76, p<0.0001 ; 25$ studies; $N=6,896$; low quality of evidence) compared to control group. The same result was not observed in patients who underwent herniorrhaphy $(\mathrm{RR}=0.86,95 \% \mathrm{CI}=0.54$, $1.36, p=0.25 ; 3$ studies; $N=1,325$; very low quality of evidence). No significant heterogeneity was observed in overall and subgroup analyses (shown in Fig. 3). Further, the symmetrical funnel plot indicated no evidence of significant publication bias.

\section{Subgroups Based on SSI Type}

Data analysis showed that AP was associated with a significant reduction in the risk of superficial SSI $(\mathrm{RR}=$ $0.56,95 \% \mathrm{CI}=0.43,0.72, p<0.0001 ; 23$ studies; $N=6,658$; low quality of evidence) compared to control group. However, in terms of deep SSI, no significant difference was observed $(\mathrm{RR}=0.70,95 \% \mathrm{CI}=0.30,1.62, p=0.40 ; 14$ studies; $N=4,638$; low quality of evidence) between AP and control groups. No significant heterogeneity was observed for both outcomes. Funnel plots for both outcomes were symmetrical, indicating no evidence of significant publication bias.

\section{Subgroups Based on Antibiotic Type}

Subgroup analysis showed that AP using amoxicillin plus clavulanic acid $(\mathrm{RR}=0.55,95 \% \mathrm{CI}=0.35,0.88, p=$ 


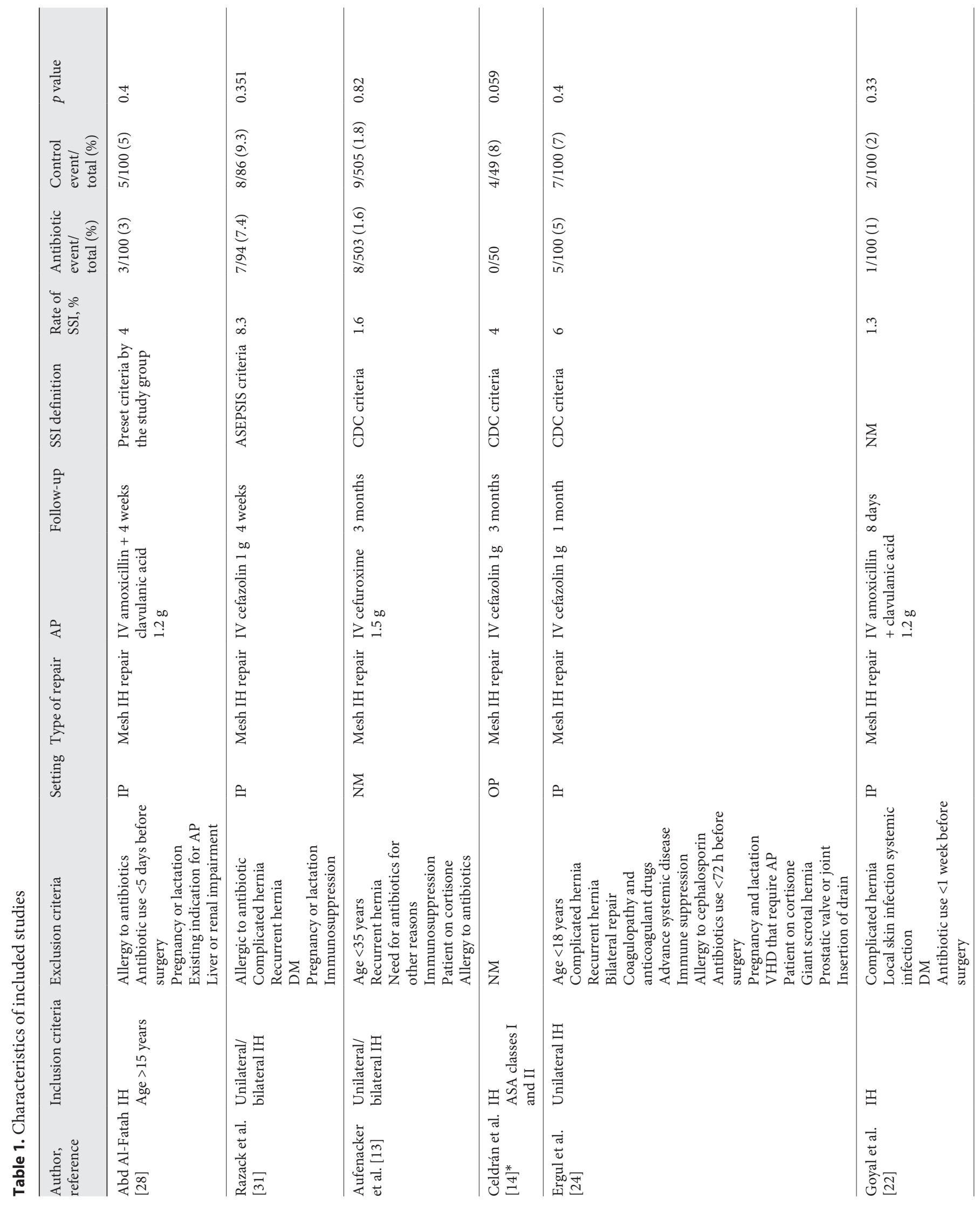




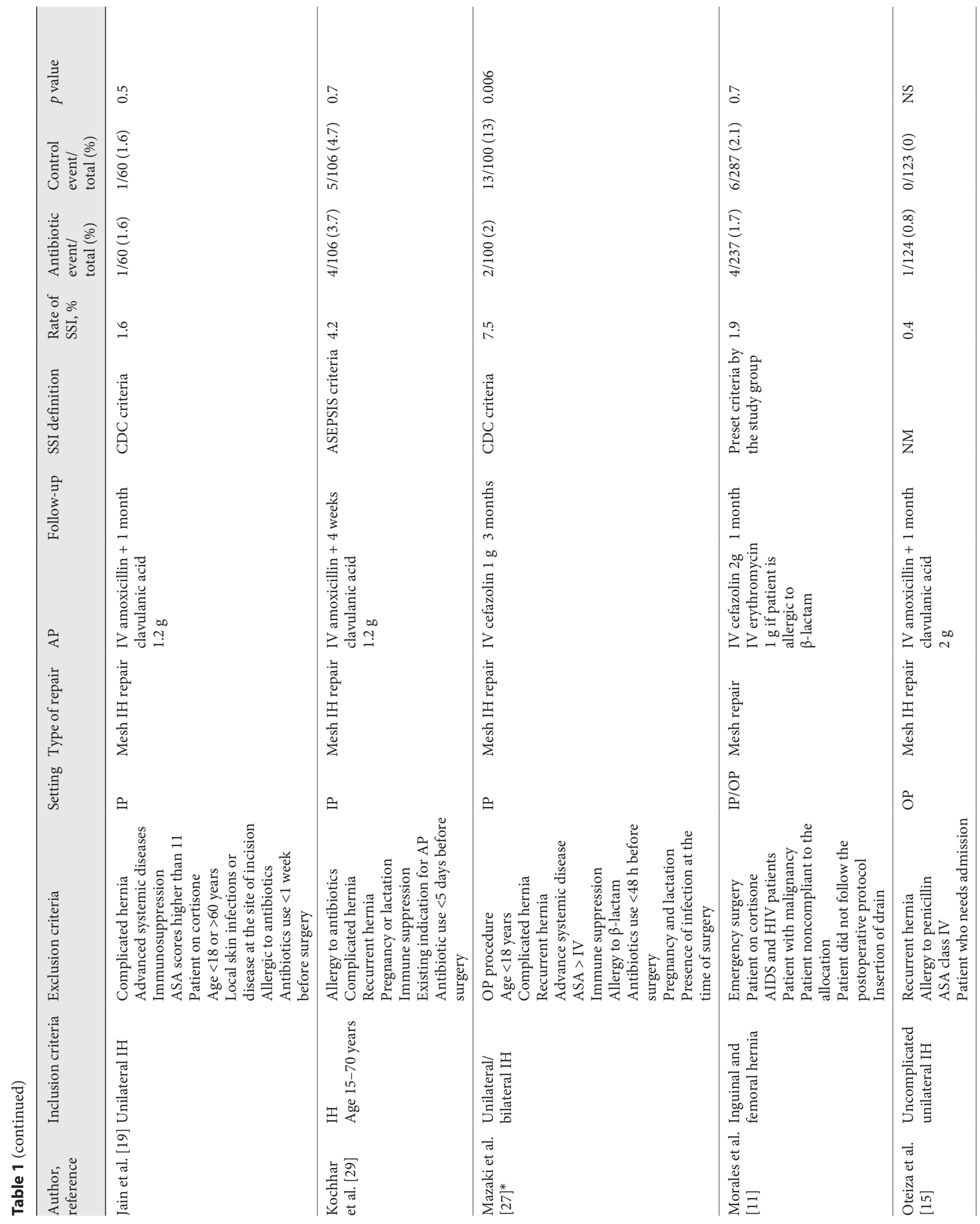




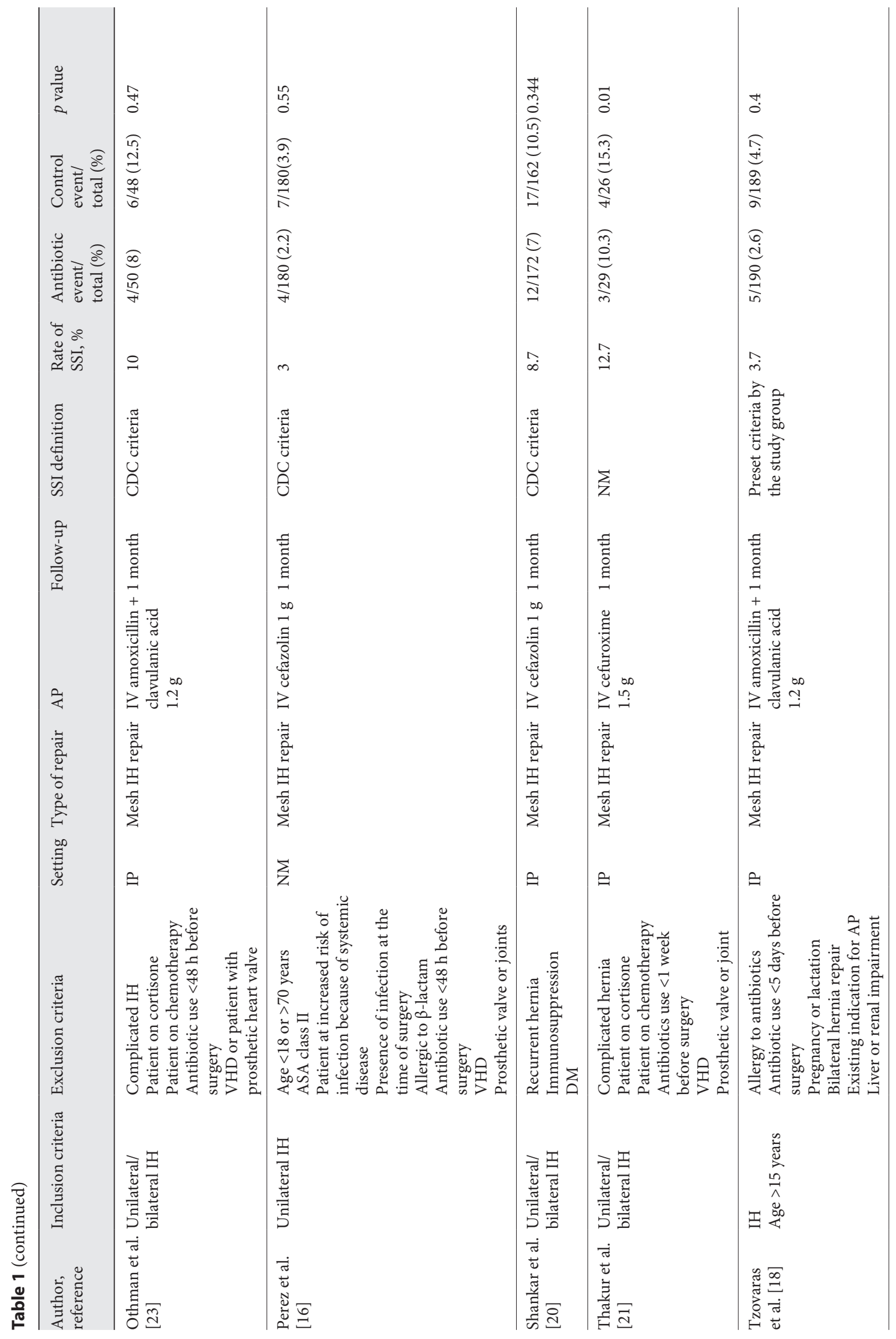




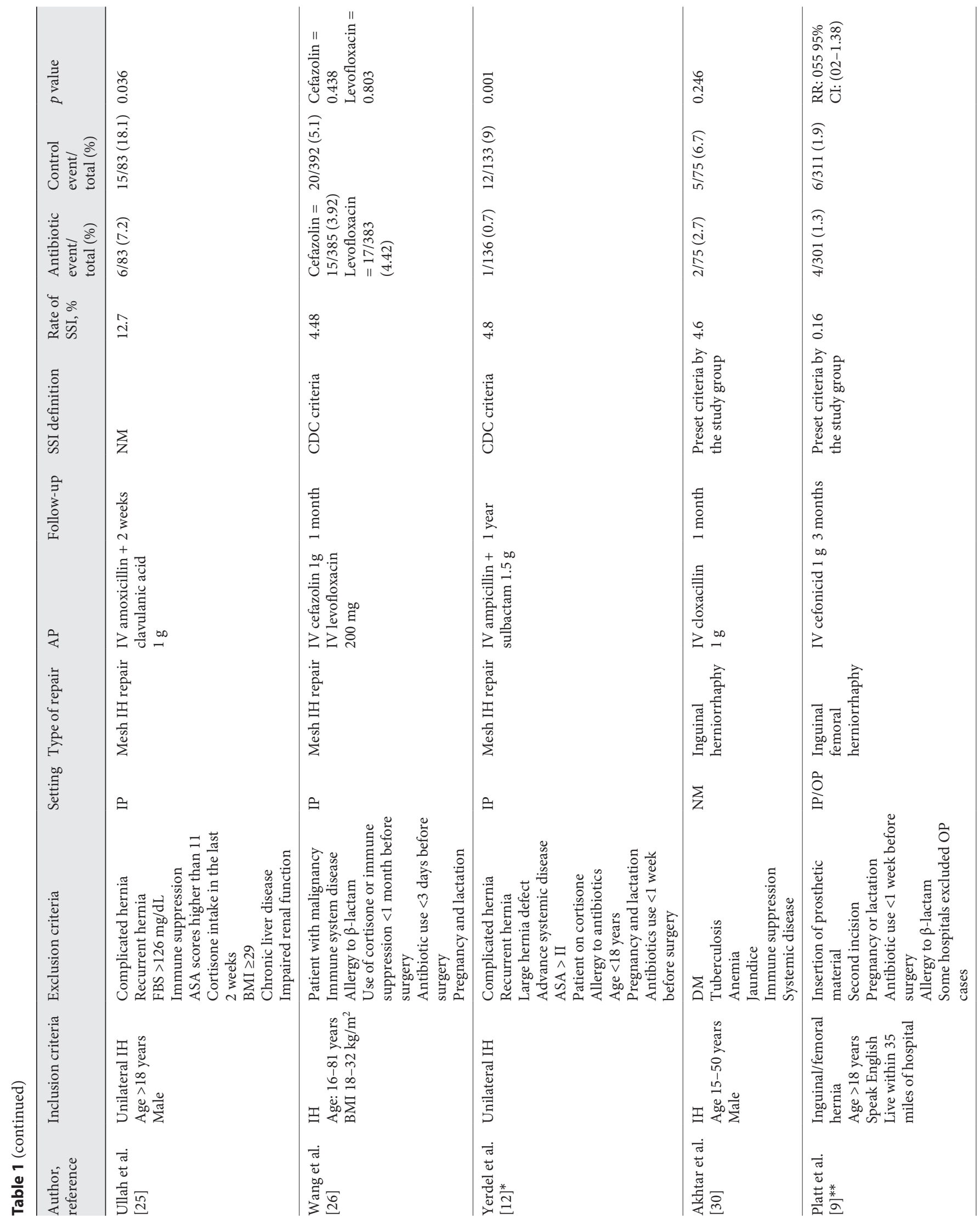




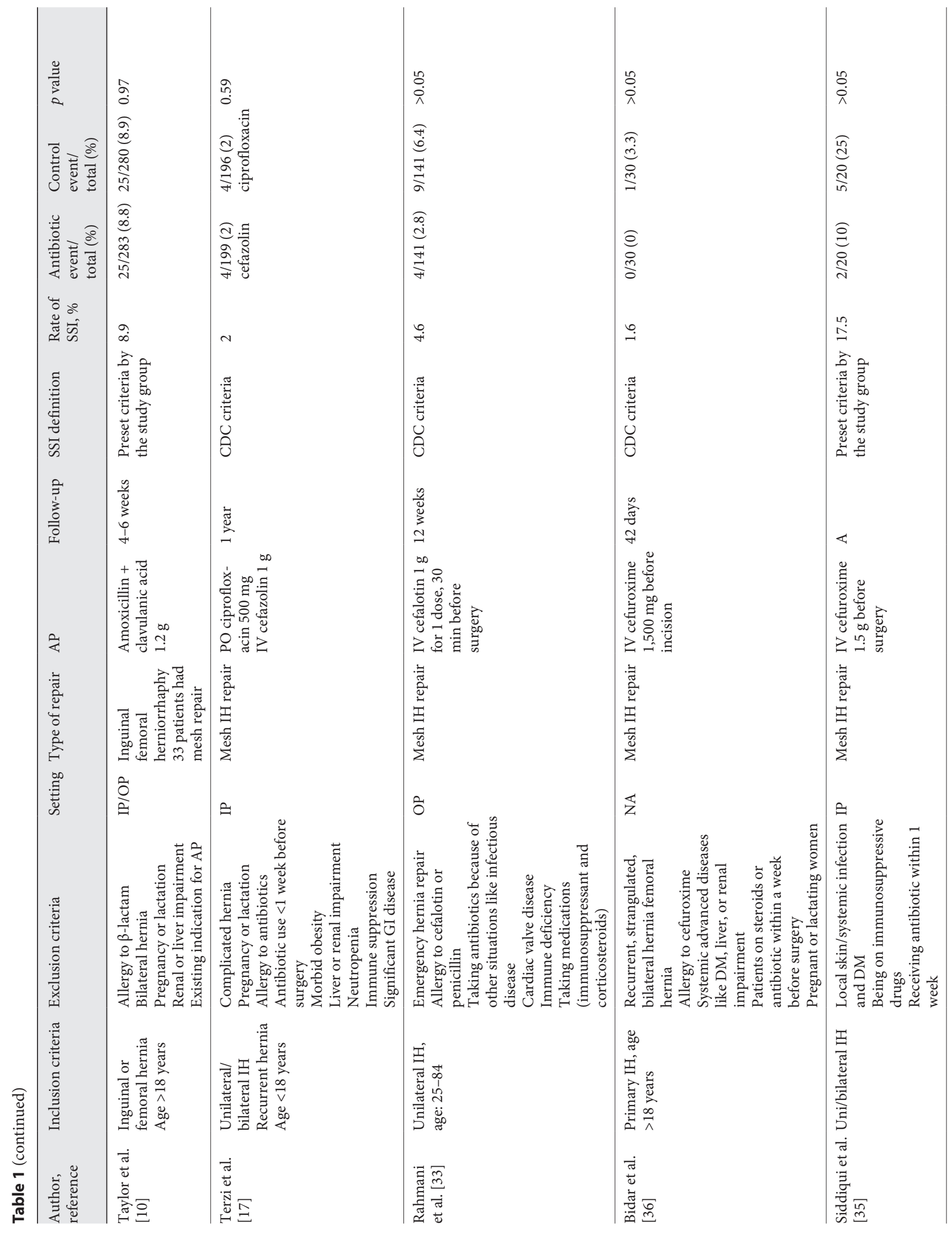




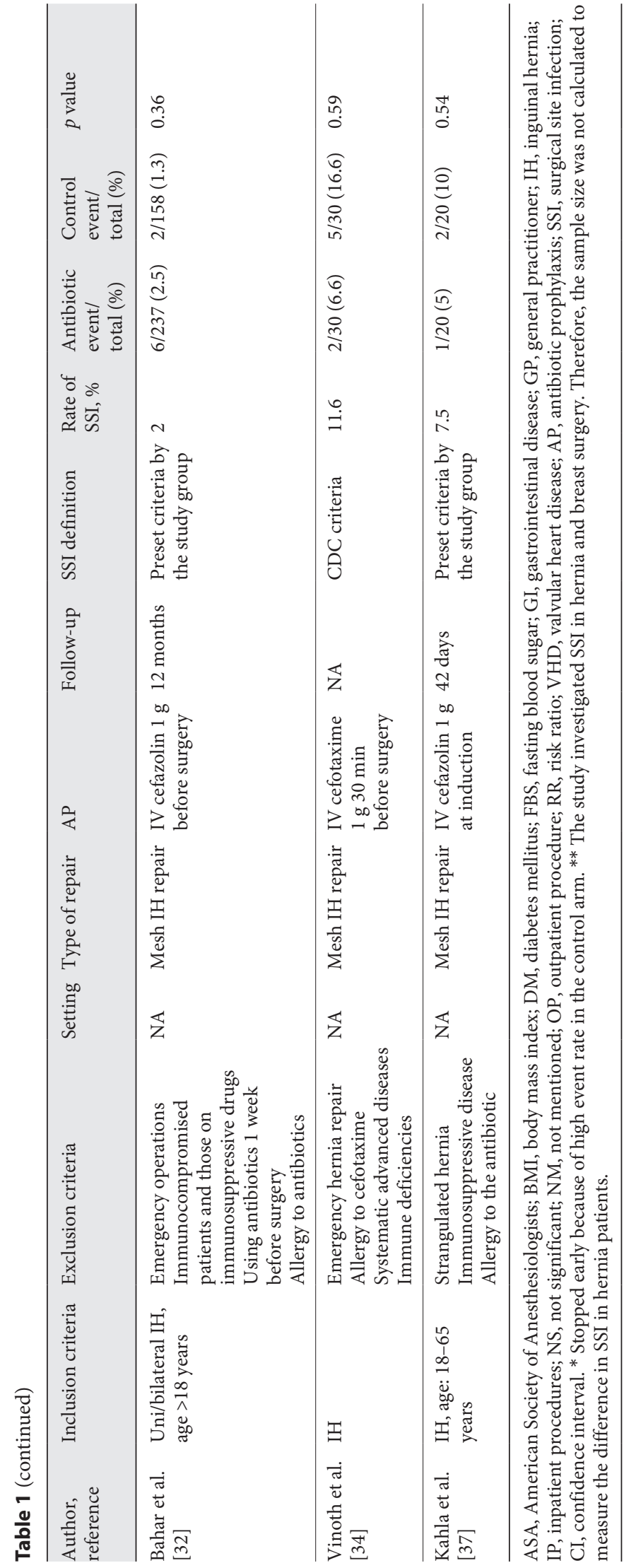

Antibiotics in Hernia Surgery
0.01 ; 9 studies; $N=1,899$; low quality of evidence) or cefazolin $(\mathrm{RR}=0.67,95 \% \mathrm{CI}=0.48,0.95, p=0.02 ; 8$ studies; $N=2553$; low quality of evidence) were associated with significant reductions in SSI risk, while prophylaxis using cefuroxime $(\mathrm{RR}=0.68,95 \% \mathrm{CI}=0.35,1.33, p=0.26 ; 4$ studies; $N=1,163$; low quality of evidence) was associated with a comparable SSI risk compared to control group. No significant heterogeneity was observed across all subgroups. An exploratory comparative analysis based on only 2 studies showed comparable risks of SSI in patients who received cephalosporins versus quinolones $(\mathrm{RR}=$ $0.90,95 \% \mathrm{CI}=0.49,1.66, p=0.74 ; 2$ studies; $N=1,169$; very low quality of evidence) $[17,26]$.

\section{Discussion}

Our meta-analysis showed that the use of AP prior to hernia surgery reduces the risk of SSI. The pooled incidence rate of SSI in the AP group was 3.5\% and 5.2\% in the no-AP group. However, subgroup analysis showed that the benefit of AP was mainly in procedures where mesh was used. The rate of SSI was not affected by choice of quinolone versus cephalosporin.

Meta-analyses exploring the same subject have reported different results [42-48]. A 2012 Cochrane review examined the role of systemic and local antibiotics in preventing SSI after hernia repair, regardless of the hernia type or procedural technique. The review demonstrated that antibiotics significantly reduced the rate of post-hernia repair SSI by $36 \%$ compared with controls but only in cases of mesh repair. However, the number needed to treat was too high to justify the regular use of AP in cases of mesh repair (NNT, 70; 95\% CI: 43-183) [43]. Evidence provided by Aufenacker et al. [46] did not support the use of AP to prevent SSI prior to mesh hernia repair.

Six other meta-analyses looked at the effects of AP prior to IH mesh repair. Those reviews [42-44, 48, 49] with one exception [47] showed AP to be beneficial in this procedure. Other factors could be related to the skill level and expertise of the performing surgeon, the procedural technique, the definition of SSI, follow-up modalities and duration, and the presence or absence of comorbidities in the study populations.

The results of observational studies looking at the use of AP prior to hernia repair are contradictory. In a single-arm prospective study that included 2002 mesh repairs of IHs, Just et al. [50] found that AP reduced the rate of SSI from 1.2 to $0.2 \%$ [50]. A similar finding was noted by Taylor et al. [51] who surveyed 2,665 IH repair 


\begin{tabular}{|c|c|c|c|c|c|c|c|}
\hline & 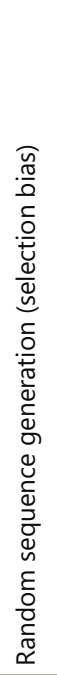 & 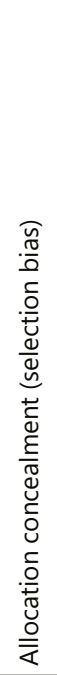 & 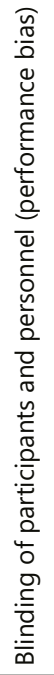 & 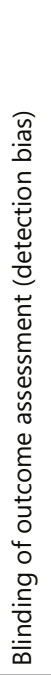 & 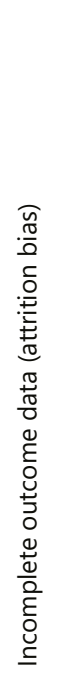 & 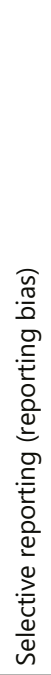 & $\begin{array}{l}\frac{n}{0} \\
\frac{.0}{0} \\
\frac{1}{d} \\
\frac{ \pm}{0}\end{array}$ \\
\hline Abd Al-Fatah 2011 & + & + & + & $?$ & + & + & + \\
\hline Abdul Razack 2015 & $?$ & + & $?$ & + & + & + & + \\
\hline Akhtar 2014 & + & $?$ & $?$ & $\theta$ & + & + & + \\
\hline Aufenacker 2004 & $\oplus$ & $?$ & + & + & + & + & + \\
\hline Bahar 2015 & $\theta$ & $\theta$ & $\theta$ & $\theta$ & + & + & $?$ \\
\hline Bidhur 2013 & + & $?$ & + & $?$ & + & + & + \\
\hline Celdran 2004 & + & $?$ & + & $?$ & 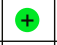 & + & $\oplus$ \\
\hline Ergul 2012 & $?$ & + & + & $\oplus$ & + & + & $\oplus$ \\
\hline Goyal 2011 & + & $\theta$ & $?$ & $\ominus$ & + & $\oplus$ & $\oplus$ \\
\hline Jain 2008 & + & $?$ & $\theta$ & $?$ & + & + & + \\
\hline Kahla 2019 & $?$ & $?$ & $\theta$ & $\ominus$ & $\oplus$ & + & $?$ \\
\hline Kochhara2014 & $?$ & $?$ & $?$ & $\oplus$ & + & + & + \\
\hline Mazaki 2014 & + & $?$ & + & + & + & + & + \\
\hline Morales 2000 & $?$ & $\theta$ & $\theta$ & $\ominus$ & $\oplus$ & + & + \\
\hline Oteiza 2003 & + & $\theta$ & $\theta$ & $?$ & + & + & + \\
\hline Othman 2011 & $\oplus$ & $?$ & $\oplus$ & $?$ & $\oplus$ & + & $\oplus$ \\
\hline Perez 2005 & + & + & + & $\oplus$ & + & + & + \\
\hline Platt 1990 & $?$ & + & + & + & $\ominus$ & + & 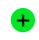 \\
\hline Rahmani 2012 & $?$ & $?$ & + & $?$ & + & $\theta$ & + \\
\hline Shankar 2010 & $?$ & + & $?$ & + & + & + & + \\
\hline Siddiqui 2017 & $\theta$ & $\theta$ & $\theta$ & $\ominus$ & $\oplus$ & $?$ & $?$ \\
\hline Taylor 1997 & $\oplus$ & $?$ & $?$ & $?$ & $?$ & + & + \\
\hline Terzi 2005 & $\oplus$ & $?$ & $?$ & $\oplus$ & $\oplus$ & 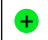 & $\oplus$ \\
\hline Thakur 2010 & $?$ & $?$ & $\theta$ & $\ominus$ & + & + & + \\
\hline Tzovaras 2006 & + & + & + & + & + & + & + \\
\hline Ulah 2013 & $?$ & $?$ & $?$ & $\ominus$ & + & + & + \\
\hline Vinoth 2015 & $?$ & $?$ & $?$ & $?$ & + & $?$ & $?$ \\
\hline Wang 2013 & + & + & $?$ & $?$ & + & + & + \\
\hline Yerdel 2001 & + & + & + & + & + & + & + \\
\hline
\end{tabular}

Fig. 2. ROB items for each study. patients and found an overall rate of SSI of $5.3 \%$. Patients who received AP had less of a risk of SSI than those who did not receive AP (4.2 vs. 7.6\%, respectively; $p=0.002)$. On the other hand, retrospective data did not support the use of AP prior to primary hernia surgery without or with mesh insertion. A series of retrospective review by Gilbert et al. [6] reported the results of 2,492 IH repairs, $74 \%$ of the patient had mesh repair, and almost half of them received AP. The group found that AP provided no additional protection against SSI in primary hernia repair irrespective of the technique. However, there was a difference in recurrent hernia mesh repair (0.43 vs. $2.2 \%)$.

After hernia repair surgery, SSI is usually managed with antibiotics and rarely requires surgical intervention or removal of the mesh which is most likely related to the fact that most SSIs are superficial $[5,52]$. However, postrepair SSI has been linked to hernia recurrence. Glassow et al. [53] described the outcome of 26,000 herniorrhaphy. They found that SSI increases the recurrence rate 4 times.

No other study linked SSI to hernia recurrence. Therefore, SSI might predispose the patient to hernia recurrence, but its role in recurrence is minor. Another factor that influences the decision to use AP is the lack of formal cost-benefit analysis. Theoretically, the use of a single dose of AP is more economical than the treatment of SSI and its possible consequences including recurrence $[9$, 54]. In hernia repair like other surgery, the choice of AP is usually driven by the micro-organism likely to have caused the infection; if there is no visceral violation, it is usually Gram-positive cocci and skin flora [52, 55], and the use of cephalosporins and penicillins appears safe. Prospective studies (were included in this analysis) comparing the use of cephalosporin versus quinolones showed no significant differences in the rate of post-hernia repair SSI $[17,26]$.

\section{Limitations}

Meta-analyses provide an assessment of the best available evidence; however, they are always limited by their primary studies. The clinical trials included in our analysis, which investigated AP prior to the use of mesh to repair uncomplicated $\mathrm{IH}$, mostly involved healthy patients and excluded patients with comorbidities. This affects the generalizability of our findings to recurrent, nonelective, or other hernia types. Other limitations of this metaanalysis are lack of unified definition for SSI and differ- 


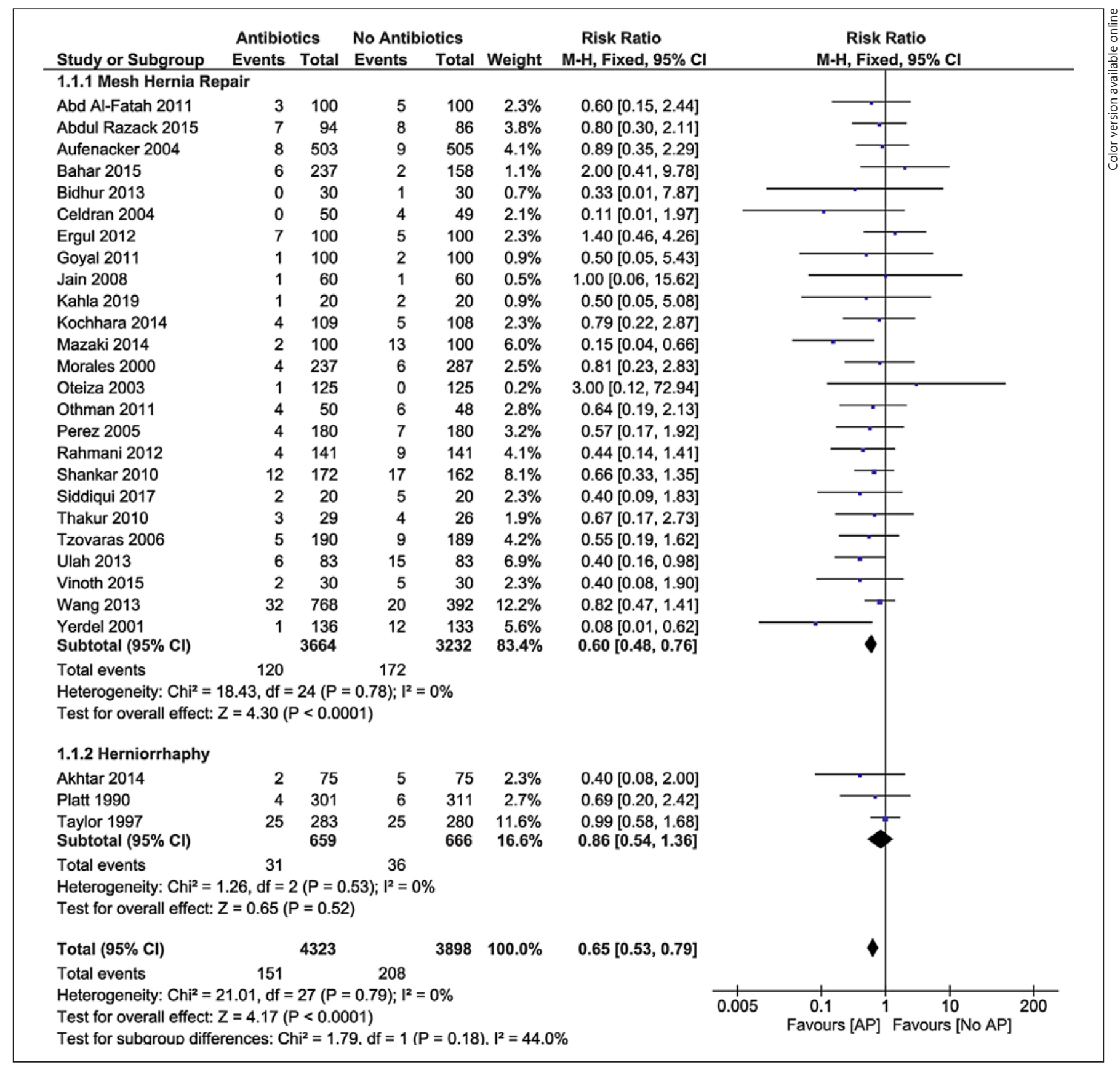

Fig. 3. Overall and subgroup analyses.

ent follow-up period/modality, which might influence the rate of SSI. Some of the studies included in our analysis had a very short follow-up; in fact, only 2 studies had 1 year follow-up: according to the WHO definition, SSI can occur up to 1 year after mesh surgery [56]. The use of different antibiotics is another constraint of our review.

Antibiotics in Hernia Surgery

\section{Conclusion}

We have demonstrated a beneficial role for systemic AP in preventing SSI after open mesh hernia repair. Our findings do not support the use of AP in cases of herniorrhaphy; however, these results should be interpreted carefully due to the low quality of available evidence, and 
further well-designed RCTs are still needed. Further, a large clinical trial is required to address issues such as cost-effectiveness, hernia recurrence, and quality of life.

\section{Acknowledgement}

H.H.B. has contributed to this work while affiliated to King Saud bin Abdulaziz University for Health Sciences, Riyadh, Saudi Arabia. The authors thank Professor Benedetta Allegranzi, Infection Prevention and Control Hub, WHO Headquarters, and Dr. Stijn de Jonge, University of Amsterdam, The Netherlands, for their expertise and assistance throughout the process.

\section{Statement of Ethics}

The King Abdullah International medical Research (KAIMRC) IRB has exempted this study from review because there was nonhuman intervention.

\section{Conflict of Interest Statement}

All the authors declare no conflicts of interest.

\section{Funding Sources}

The authors did not receive any funding for this study.

\section{Author Contributions}

L.A.R., W.M., and N.F.: contributed to article screening, data extraction, and risk of bias assessment; H.B. data analysis; M.F. and A.A.: idea conception and supervision. All the authors revised the final version of the manuscript and approved it for publication.

\section{References}

1 World Health Organization. Surgical site infections; 2020.

2 Daniels IR, Smart NJ. In support of mesh for hernia repair. Br J Surg. 2019;106(7):815-6.

3 Tubre DJ, Schroeder AD, Estes J, Eisenga J, Fitzgibbons RJ. Surgical site infection: the "Achilles Heel" of all types of abdominal wall hernia reconstruction. Hernia. 2018;22(6): 1003-13.

4 Ashrafi D, Siddaiah-Subramanya M, Memon B, Memon MA. Causes of recurrences after open inguinal herniorrhaphy. Hernia. 2019; 23(4):637-45.

5 Kark AE, Kurzer MN, Belsham PA. Three thousand one hundred seventy-five primary inguinal hernia repairs: advantages of ambulatory open mesh repair using local anesthesia. J Am Coll Surg. 1998;186(4):447-56.

6 Gilbert AI, Felton LL. Infection in inguinal hernia repair considering biomaterials and antibiotics. Surg Gynecol Obstet. 1993; 177(2): 126

7 Bailey IS, Karran SE, Toyn K, Brough P, Ranaboldo C, Karran SJ. Community surveillance of complications after hernia surgery. BMJ. 1992;304(6825):469-71.

8 HerniaSurge Group. International guidelines for groin hernia management. Hernia. 2018; 22(1): 1165

9 Platt R, Zaleznik DF, Hopkins CC, Dellinger EP, Karchmer AW, Bryan CS, et al. Perioperative antibiotic prophylaxis for herniorrhaphy and breast surgery. N Engl J Med. 1990; 322(3):153-60.

10 Taylor EW, Byrne DJ, Leaper DJ, Karran SJ, Browne MK, Mitchell KJ. Antibiotic prophylaxis and open groin hernia repair. World J Surg. 1997;21(8):811-5.

11 Morales R, Carmona A, Pagán A, GarcíaMenéndez C, Bravo R, Hernández M, et al.
Utilidad de la profilaxis antibiótica en la reducción de la infección de herida en la reparación de la hernia inguinal o crural mediante malla de polipropileno. Cir Esp. 2000;67(1): 519.

12 Yerdel MA, Akin EB, Dolalan S, Turkcapar AG, Pehlivan M, Gecim IE, et al. Effect of single-dose prophylactic ampicillin and sulbactam on wound infection after tension-free inguinal hernia repair with polypropylene mesh: the randomized, double-blind, prospective trial. Ann Surg. 2001;233(1):26.

13 Aufenacker TJ, van Geldere D, Van Mesdag T, Bossers AN, Dekker B, Scheijde E, et al. The role of antibiotic prophylaxis in prevention of wound infection after Lichtenstein open mesh repair of primary inguinal hernia: a multicenter double-blind randomized controlled trial. Ann Surg. 2004;240(6):955-1.

14 Celdrán A, Frieyro O, de la Pinta JC, Souto JL, Esteban J, Rubio JM, et al. The role of antibiotic prophylaxis on wound infection after mesh hernia repair under local anesthesia on an ambulatory basis. Hernia. 2004;8(1):20-2

15 Oteiza F, Ángel Ciga M, Ortiz H. Profilaxis antibiótica en la hernioplastia inguinal. Cirugía Española. 2004;75(2):69-71.

16 Perez AR, Roxas MF, Hilvano SS. A randomized, double-blind, placebo-controlled trial to determine effectiveness of antibiotic prophylaxis for tension-free mesh herniorrhaphy. J Am Coll Surg. 2005;200(3):393-8.

17 Terzi C, Kiliç D, Ünek T, Hoşgörler F, Füzün M, Ergör G. Single-dose oral ciprofloxacin compared with single-dose intravenous cefazolin for prophylaxis in inguinal hernia repair: a controlled randomized clinical study. J Hosp Infect. 2005;60(4):340-7.

18 Tzovaras G, Delikoukos S, Christodoulides G, Spyridakis M, Mantzos F, Tepetes K, et al. The role of antibiotic prophylaxis in elective tension-free mesh inguinal hernia repair: results of a single-centre prospective randomised trial. Int J Clin Prac. 2007;61(2):236-9.

19 Jain SK, Jayant M, Norbu C. The role of antibiotic prophylaxis in mesh repair of primary inguinal hernias using prolene hernia system: a randomized prospective double-blind control trial. Trop Doct. 2008;38(2):80-2.

20 Shankar VG, Srinivasan K, Sistla SC, Jagdish S. Prophylactic antibiotics in open mesh repair of inguinal hernia: a randomized controlled trial. Int J Surg. 2010;8(6):444-7.

21 Thakur L, Upadhyay S, Peters NJ, Saini N, Deodhar M. Prophylactic antibiotic usage in patients undergoing inguinal mesh hernioplasty: a clinical study. Indian J Surg. 2010;72(3):240-2.

22 Goyal A, Garg R, Jenaw RK, Jindal DK. Role of prophylactic antibiotics in open inguinal hernia repair: a randomised study. Indian J Surg. 2011;73(3):190-3.

23 Othman I. Prospective randomized evaluation of prophylactic antibiotic usage in patients undergoing tension free inguinal hernioplasty. Hernia. 2011;15(3):309-13.

24 Ergul Z, Akinci M, Ugurlu C, Kulacoglu H, Yilmaz KB. Prophylactic antibiotic use in elective inguinal hernioplasty in a trauma center. Hernia. 2012;16(2):145-51.

25 Ullah B, Khan SA, Ahmed S, Pasha T. Efficacy of preoperative single dose antibiotic in patients undergoing mesh repair for inguinal hernia. J Ayub Med Coll Abbottabad. 2013; 25(1-2):103-5

26 Wang J, Ji G, Yang Z, Xi M, Wu Y, Zhao P, et al. Prospective randomized, double-blind, placebo controlled trial to evaluate infection prevention in adult patients after tension-free inguinal hernia repair. Int J Clin Pharmacol Ther. 2013;51(12):924-31. 
27 Mazaki T, Mado K, Masuda H, Shiono M, Tochikura N, Kaburagi M. A randomized trial of antibiotic prophylaxis for the prevention of surgical site infection after open mesh-plug hernia repair. Am J Surg. 2014;207(4):476-84.

28 Abd Al-Fatah M. The role of antibiotic prophylaxis in prevention of wound infection after Lichtenstein repair of primary inguinal hernia. Al-Azhar Assiut Med J. 2011;9:173-80.

29 Kochhar G, Andley M, Saha S, Kumar A, Saurabh G, Kumar A, et al. Randomized controlled trial to evaluate the role of antibiotic prophylaxis in lichtenstein hernia repair. J Young Med Res. 2014;1(2):1-8.

30 Akhtar K, Khan Z, Bhatti AM, Mahmood K. Impact of using prophylactic antibiotic on prevention of wound infection in inguinal herniorrhaphy. Pak Armed Forces Med J. 2015;65(4):453-7.

31 Razack A, Kapoor KK, Tambat RM. The role of single dose antibiotic prophylaxis in open mesh repair of inguinal hernia: a prospective, double blind randomized trial. J Evol Med Dent Sci. 2015;4(35):6017-27.

32 Bahar MM, Nooghabi AJ, Nooghabi MJ, Jangjoo A. The role of prophylactic cefazolin in the prevention of infection after various types of abdominal wall hernia repair with mesh. Asian J Surg. 2015;38(3):139-44.

33 Rahmani N, Sarav K, Sayadi M, Khalilia A. Effect of prophylactic antibiotics in preventing wound infection in hernioplasty with inguinal mesh in Lichtenstein method. J Mazandaran Univ Med Sci. 2012;22(90):88-93.

34 Vinoth N, Karthikeyan CRM, Parmar H. Role of antibiotic prophylaxis in open inguinal hernioplasty: a prospective randomized clinical trial. Int Arch Integ Med. 2015;2(3):57-67.

35 Siddiqui MAR, Aleem MA, Rahman MS, Zafreen F. Role of prophylactic antibiotics in open mesh inguinal hernia repair. J Armed Forces Med Coll. 2019;13(2):18-21.

36 Bidar KC, Regmi R, Agarwal CS, Pathania OP. Role of single dose antibiotic prophylaxis in prevention of wound infection following lichtenstein inguinal hernioplasty: a randomized clinical trial. Nepal J Med Sci. 2013;2(2):10813.
37 Kahla S, Gaber A, Al-Rahawy M. The role of antibiotic prophylaxis in the prevention of surgical- site infection after hernioplasty in Menoufia University Hospital. Menoufia Med J. 2019;32(3):1137-41.

38 Higgins JP, Thomas J, Chandler J, Cumpston M, Li T, Page M, et al. Cochrane handbook for systematic reviews of interventions. John Wiley \& Sons; 2019.

39 Moher D, Liberati A, Tetzlaff J, Altman DG Preferred reporting items for systematic reviews and meta-analyses: the PRISMA statement. BMJ. 2009;339(7):b2535.

40 Higgins JP, Altman DG, Gøtzsche PC, Jüni P, Moher D, Oxman AD, et al. The Cochrane Collaboration's tool for assessing risk of bias in randomised trials. BMJ. 2011;343:d5928.

41 Schünemann HJ, Oxman AD, Brozek J, Glasziou P, Bossuyt P, Chang S, et al. GRADE: assessing the quality of evidence for diagnostic recommendations. Evid Based Med. 2008; 13(6):162-3.

42 Li JF, Lai DD, Zhang XD, Zhang AM, Sun KX, Luo HG, et al. Meta-analysis of the effectiveness of prophylactic antibiotics in the prevention of postoperative complications after tension-free hernioplasty. Can J Surg. 2012; 55(1):27.

43 Sanchez-Manuel FJ, Seco-Gil JL. Antibiotic prophylaxis for hernia repair. Cochrane $\mathrm{Da}-$ tabase Syst Rev. 2004;4:CD003769.

44 Sanabria A, Domínguez LC, Valdivieso E, Gómez G. Prophylactic antibiotics for mesh inguinal hernioplasty: a meta-analysis. Ann Surg. 2007;245(3):392.

45 Yin Y, Song T, Liao B, Luo Q, Zhou Z. Antibiotic prophylaxis in patients undergoing open mesh repair of inguinal hernia: a metaanalysis. Am Surg. 2012;78(3):359-65.

46 Aufenacker TJ, Koelemay MJ, Gouma DJ, Simons MP. Systematic review and meta-analysis of the effectiveness of antibiotic prophylaxis in prevention of wound infection after mesh repair of abdominal wall hernia. $\mathrm{Br} J$ Surg. 2006;93(1):5-10.
47 Erdas E, Medas F, Pisano G, Nicolosi A, Calò PG. Antibiotic prophylaxis for open mesh repair of groin hernia: systematic review and meta-analysis. Hernia. 2016;20(6): 765-76.

48 Mazaki T, Mado K, Masuda H, Shiono M Antibiotic prophylaxis for the prevention of surgical site infection after tension-free hernia repair: a Bayesian and frequentist metaanalysis. J Am Coll Surg. 2013;217(5):7884.

49 Boonchan T, Wilasrusmee C, McEvoy M, Attia J, Thakkinstian A. Network meta-analysis of antibiotic prophylaxis for prevention of surgical-site infection after groin hernia surgery. Br J Surg. 2017;104(2):e106.

50 Just E, Botet X, Martínez S, Escolà D, Moreno I, Duque E. Reduction of the complication rate in Liechtenstein hernia repair. Int J Surg. 2010;8(6):462-5.

51 Taylor EW, Duffy K, Lee K, Hill R, Noone A Macintyre I, et al. Surgical site infection after groin hernia repair. Br J Surg. 2004;91(1): 105-11.

52 Riley KD, Lilly MC, Arregui ME. Management of complications following inguinal hernia repair. Probl Gen Surg. 2002;19(4):97108.

53 Glassow F. Is postoperative wound infection following simple inguinal herniorrhaphy a predisposing cause of recurrent hernia? Can Med Assoc J. 1964;91(16):870-1.

54 Davey PG, Nathwani D. What is the value of preventing postoperative infections? New Horiz. 1998;6(2 Suppl 1):S64-71.

55 Weed HG. Antimicrobial prophylaxis in the surgical patient. Med Clin North Am. 2003; 87(1):59-75.

56 World Health Organization. Protocol for surgical site infection surveillance with a focus on settings with limited resources. 2018. Available from: https://www.who.int/infection-prevention/tools/surgical/SSI- surveillance-protocol.pdf?ua=1 Accessed 2020 Aug 20. 